December 15, 2020

\title{
Palmitoylation of KChIP3 controls baseline mucin secretion
}

\author{
Cantero-Recasens $\mathrm{G}^{1,2, *}$, Burballa $\mathrm{C}^{1,2}$, Duran $\mathrm{M}^{1}$, Brouwers $\mathrm{N}^{2}$, Malhotra $\mathrm{V}^{2,3, *}$.
}

Affiliations:

${ }^{1}$ Renal Physiopathology Group, Vall d'Hebron Research Institute (VHIR), Pg. Vall d'Hebron 119-129, 08035 Barcelona, Spain

${ }^{2}$ Centre for Genomic Regulation (CRG), The Barcelona Institute for Science and Technology, Dr. Aiguader 88, 08003 Barcelona, Spain

${ }^{3}$ Institució Catalana de Recerca i Estudis Avançats (ICREA), Pg. Lluis Companys 23, 08010 Barcelona, Spain

* Correspondence to:

Gerard Cantero-Recasens, Renal Physiopathology Group, Vall d'Hebron Research Institute (VHIR), Pg. Vall d'Hebron 119-129, 08035 Barcelona, Spain

E-mail address: gerard.cantero@vhir.org

Vivek Malhotra, Department of Cell and Developmental Biology, Centre for Genomic Regulation (CRG), Carrer Dr. Aiguader 88, 08003 Barcelona, Spain

E-mail address: vivek.malhotra@ crg.eu

\section{Abstract}

Baseline mucin secretion (BMS) is independent of external agonists and controlled by a small calcium binding protein named KChIP3. KChIP3-hosting mucin granules are not released until intracellular cytosolic calcium oscillations reach a threshold, KChIP3 binds calcium and detaches from granules, allowing their fusion to plasma membrane. Loss of KChIP3 or blocking its membrane attachment causes mucin hypersecretion. How is KChIP3 recruited to mucin granules? We show here that zDHHC (aspartate-histidine-histidine-cysteine motif in a cysteine-rich, zinc fingerlike domain) S-acyl-transferase dependent palmitoylation modulates binding of KChIP3 to mucin granules thereby affecting mucin secretion. We have found that inhibiting zDHHC-mediated palmitoylation in differentiated HT29-18N2, which express the Golgi-localized zDHHC3 and zDHHC4, releases KChIP3 from mucin granules and increases baseline mucin secretion. Mutation of the palmitoylation sites in KChIP3 (Cysteines 122 and 123 to Alanine) quantitatively reduces its attachment to mucin granules. Expression of KChIP3-WT in HT29-18N2 cell lines stably depleted of KChIP3 inhibits mucin secretion, whereas expression of nonpalmitoylated KChIP3 (KChIP3-AA) only partially rescues the effect of KChIP3 depletion and the cells maintain higher levels of baseline secretion compared to KChIP3-WT cells. Altogether, our data suggest that zDHHC3 or zDHHC4-dependent palmitoylation is involved in KChIP3 recruitment to mucin granules to control the baseline mucin secretion.

$\underline{\text { Keywords }}$

KChIP3, zDHHC-dependent palmitoylation, mucin granules, baseline mucin secretion, goblet cells 


\section{$\underline{\text { Introduction }}$}

The mucus layer, which protects the respiratory and gastrointestinal systems from pathogens, toxins and allergens (Cone, 2009), is mainly composed of ions, water and mucins that are produced and secreted by specialized goblet cells (Birchenough et al, 2015). To date, five gel-forming mucins have been described in humans, including MUC2 as the main mucin for the intestine and colon (Thornton et al, 2008). Gelforming mucins are heavily glycosylated during their traffic across the Golgi apparatus. These highly glycosylated mucins are then packed into specialized micrometer-sized granules, which undergo a maturation process that results in condensation of mucins. Mature granules, containing condensed mucins, fuse to apical plasma membrane by a $\mathrm{Ca}^{2+}$ dependent process in the absence of extracellular agonist (Baseline Mucin Secretion or BMS) or upon external agonist stimulation (Stimulated Mucin Secretion or SMS) (Adler et al, 2013; Thornton et al, 2008). It is suggested that baseline secretion is the main mode of mucin release in healthy tissue and contribute to mucus layer maintenance; whereas stimulated secretion is more important in allergic and infectious responses (Zhu et al, 2015). Changes in the levels of mucins secreted by the baseline or stimulated secretion can lead to bacterial invasion, inflammation and consequently to airway or gastrointestinal pathologies (Fahy \& Dickey, 2010; Pelaseyed et al, 2014; Evans \& Koo, 2009).

We have previously reported that stimulated secretion requires extracellular calcium entry through the cooperation of sodium channels (TRPM4/TRPM5) and sodiumcalcium exchangers (NCXs) (Cantero-Recasens et al, 2019), whereas baseline secretion depends on intracellular calcium oscillations and a small calcium binding protein called KChIP3 (Cantero-Recasens et al, 2018). Our data indicate that KChIP3 is the high-affinity $\mathrm{Ca}^{2+}$ sensor that negatively regulates baseline mucin secretion in colonic goblet cells (Cantero-Recasens et al, 2018). Briefly, KChIP3 is recruited to mucin granules and these granules bearing KChIP3 do not fuse. When cytosolic calcium reaches a certain threshold, mainly due to ryanodine receptor-mediated calcium oscillations, KChIP3 binds calcium, consequently being released from the granules, which then go on to release their contents by fusion to plasma membrane. But how is the cytosolic KChIP3 recruited to granules? Interestingly, KChIP3 is known to be palmitoylated, a post-translational modification (PTM) that enhances its location at the plasma membrane in neurons (Takimoto et al, 2002). Palmitoylation of KChIP3 could, thus, be a mechanism for its recruitment to mucin granules in goblet cells.

Palmitoylation is a reversible PTM that involves the attachment of fatty acids (predominantly palmitic acid) to cysteine residues. This PTM has a major role in the regulation of trafficking (and function) of modified proteins (Lemonidis et al, 2014). Palmitoylation in mammals is catalyzed by a family of 24 zDHHC S-acyl-transferase enzymes (Fukata et al, 2004) that contain a conserved 51-aminoacid zDHHC-CR domain (aspartate-histidine-histidine-cysteine motif in a cysteine-rich, zinc finger domain). Several studies have reported the location of many zDHHCs along the compartments of the secretory pathway (Ernst et al, 2018; Rocks et al, 2010).

Here we demonstrate that palmitoylation by zDHHC S-acyl transferase Golgi enzymes is necessary for KChIP3 recruitment to mucin granules. The description of our results follows. 


\section{$\underline{\text { Results }}$}

1. Inhibiting palmitoylation prevents KChIP3 function in HT29-18N2 cells

To assess whether palmitoylation controls KChIP3 recruitment to mucin granules and its function as a negative regulator of baseline mucin secretion, we used 2bromopalmitate (2-BP), which is a known inhibitor of zDHHC-dependent palmitoylation (Jennings et al, 2009). HT29-18N2 cell line stably expressing KChIP3 tagged with GFP at the C-terminus (KChIP3-GFP) (described in Cantero-Recasens et $a l, 2018)$ and respective control cells were differentiated for 6 days. We first measured MUC5AC secretion in both cell lines treated with vehicle or $100 \mathrm{nM} 2-\mathrm{BP}$ in the absence (baseline) or presence (stimulated) of the physiological stimulus ATP $\left(100 \mu \mathrm{M}\right.$ in a solution containing $1.2 \mathrm{mM} \mathrm{CaCl}_{2}$ ). After $30 \mathrm{~min}$ at $37^{\circ} \mathrm{C}$, extracellular medium was collected and dot blotted with anti-MUC5AC antibody as described previously (Mitrovic et al, 2013). Our results confirmed that KChIP3 overexpression (KChIP3-GFP cell line) reduced baseline secretion compared to control cells without affecting stimulated secretion (35\% reduction). Importantly, treatment with 2-BP further increased baseline MUC5AC secretion in both control and KChIP3-GFP differentiated cells $(50 \%$ and $30 \%$ increase respectively, compared to vehicle treated control cells) without affecting stimulated secretion (Figure 1A).

We tested KChIP3 recruitment to MUC5AC granules using differentiated KChIP3GFP cells. KChIP3-GFP cells were treated with vehicle or $100 \mathrm{nM}$ 2-BP for 30 minutes at $37^{\circ} \mathrm{C}$, then permeabilized and washed extensively to remove the soluble cytoplasmic pool of KChIP3 as described previously (Cantero-Recasens et al, 2018). Our data show that a fraction of KChIP3 signal colocalizes with a pool of MUC5ACcontaining granules, which is completely abolished after treatment with 2-BP (Manders' coefficient $=0.19$ vs 0.0 , respectively, $\mathrm{p}=0.02)$ (Figure 1B, quantification in Figure 1C).

These data reveal that inhibition of palmitoylation affects KChIP3 recruitment to MUC5AC containing granules and enhances baseline secretion.

Next, we checked which of the human zDHHC palmitoylation enzymes are expressed in differentiated goblet cells and therefore could be the target of 2-BP inhibition. We used available on-line tools (i.e. Uniprot database, the expression atlas from EMBL$\mathrm{EBI}$, and the proteome abundance atlas of 29 healthy human tissues (Wang et al, 2019)) to discard those zDHHC enzymes not localized at the Golgi apparatus, where we suggest KChIP3 might be recruited (Cantero-Recasens et al, 2018), and not expressed in mucin-producing tissues like the colon or lungs. This analysis revealed that only eight zDHHC palmitoyltransferases (out of 24 zDHHC present in humans) are found at the Golgi apparatus and expressed in the colon and/or lungs (Figure S1A). Then, we extracted RNA from undifferentiated and differentiated HT29-18N2 cells (as described previously (Cantero-Recasens et al, 2018)) and tested their expression. Our results revealed that zDHHC2, zDHHC3, zDHHC4, zDHHC13, zDHHC14and zDHHC17 are expressed in HT29-18N2 cells (Figure S1B), but only zDHHC3 and zDHHC4 are upregulated in differentiated HT29-18N2 cells (4 and 3.5 fold increase, respectively) similar to KChIP3 behaviour (Cantero-Recasens et al, 2018), while zDHHC2, zDHHC13, zDHHC14 and zDHHC17 levels are reduced after differentiation (Figure S1C). 


\section{Mutation of KChIP3 palmitoylation residues reduces its recruitment to mucin granules}

Previous studies have demonstrated that KChIP3 cysteines 122 and 123 are palmitoylated (Takimoto et al, 2002). Thus, to better assess the role of palmitoylation on KChIP3 function, we generated a KChIP3-AA (rAA) construct where both cysteines are mutated to alanines (Figure 2A). We generated HT29-18N2 cells stably overexpressing KChIP3-WT (rWT) or KChIP3-AA (rAA) (shRNA-targeted sequence of both constructs was mutated to be resistant to KCNIP3 shRNA) in a KChIP3-KD background to avoid contribution of the endogenous KChIP3 because KChIP3 is known to form dimers (Osawa et al, 2005). To confirm the knock-down (KD) efficiency and expression of the rescue constructs, RNA was extracted from differentiated control, KChIP3-KD, KChIP3-GFP, rWT and rAA HT29-18N2 cells and the levels of endogenous and overexpressed KCNIP3 monitored by qPCR. KChIP3-KD, rWT and rAA cells showed greater than $70 \%$ reduction in KCNIP3 endogenous mRNA levels compared to control and KChIP3-GFP cells (90\%, 72\% and $80 \%$, respectively). In addition, KChIP3-GFP, rWT and rAA showed a significant increase in exogenous KCNIP3 levels compared to control cells (Figure 2B). Overexpression of KChIP3 (tagged with GFP) was further confirmed by western blot for rescue cell lines rWT and rAA (Figure 2C).

These cell lines were then used to test whether KChIP3-AA, which cannot be palmitoylated (Takimoto et al, 2002), was recruited to mucin granules (CanteroRecasens et al, 2018). Rescue cells lines expressing KChIP3-WT (rWT) or KChIP3AA (rAA) were differentiated and seeded on glass-bottom plates to allow maximum polarization. After differentiation, rWT and rAA cells were treated with vehicle or $100 \mathrm{nM} 2-\mathrm{BP}$ for 30 minutes at $37^{\circ} \mathrm{C}$, and were then permeabilized and washed extensively to remove the soluble cytoplasmic pool of KChIP3, as described before (Cantero-Recasens et al, 2018). Immunofluorescence microscopy with anti-KChIP3 antibody and anti-MUC5AC antibody showed characteristic KChIP3 punctae that partially colocalized with MUC5AC in rWT cells (Figure 3A, upper-left panel), which was significantly reduced in 2-BP treated cells (Figure 3A, lower-left panel) (Manders' coefficient $=0.12$ vs 0.05 , respectively, $\mathrm{p}<0.05$ ) (Figure 3B). Interestingly, although rAA cells also showed moderate numbers of KChIP3 punctae (Figure 3A, upper-right panel), they did not colocalize with MUC5AC and these numbers were not affected by 2-BP treatment (Figure 3A, lower-right panel) (Manders' coefficient $=0.05$ vs 0.05, respectively, n.s.) $($ Figure 3B) .

\section{KChIP3 palmitoylation mutant does not inhibit baseline mucin secretion}

Does inhibiting association of KChIP3 palmitoylation mutant (KChIP3-AA) to mucin granules affect KChIP3 function as a brake for baseline mucin secretion? To answer this question, we have measured MUC2 and MUC5AC baseline secretion in control, KChIP3-KD, rWT and rAA differentiated HT29-18N2 cells as described previously (Cantero-Recasens et al, 2019). Briefly, after $30 \mathrm{~min}$ at $37^{\circ} \mathrm{C}$, extracellular medium was collected and dot blotted with anti-MUC5AC or anti-MUC2 antibody. Our results confirm that depletion of KChIP3 (KChIP3-KD cells) leads to an increase in both MUC2 and MUC5AC baseline secretion (2-fold increase for both mucins) (Figure 3C and 3D). Importantly, overexpression of KChIP3-WT (rWT cells) completely rescued the increase in secretion phenotype and blocked baseline secretion (70\% 
reduction in MUC2 and 80\% reduction in MUC5AC, compared to control cells). In contrast, overexpression of KChIP3 palmitoylation mutant (rAA cells) only partially rescued the KD phenotype and did not further inhibit baseline secretion (Figure 3C and 3D).

\section{$\underline{\text { Discussion }}$}

The high-affinity $\mathrm{Ca}^{2+}$ binding protein KChIP3 (potassium voltage-gated channel interacting protein 3), also known as DREAM and Calsenilin (Carrión et al, 1999; Buxbaum et al, 1998), is a multifunctional protein that localizes to a pool of mucin granules in goblet cells to control their release propensity in the absence of external stimuli. Previous studies revealed that KChIP3 responds to intracellular calcium levels, which promotes its disassociation from granules to promote mucin secretion (Cantero-Recasens et al, 2018). But how is KChIP3 recruited to mucin granules? In neurons, KChIP3 can be found at the plasma membrane forming a complex with voltage-gated potassium channels (e.g. Kv4.3) to regulate their activity. Interestingly, it was shown that palmitoylation of KChIP3 at cysteines 47 and 48 was required for efficient plasma membrane localization and enhancement of ion channel activity (Takimoto et al, 2002). Palmitoylation is a reversible post-translational modification that contributes to and stabilizes the association of proteins with cellular membranes (Lemonidis et al, 2014). This prompted us to test whether palmitoylation of KChIP3 affects its recruitment to mucin granules

Our data reveal that zDHHC-dependent palmitoylation (possibly by zDHHC3 or zDHHC4) is necessary for KChIP3 function as a negative regulator of baseline mucin secretion. Blocking KChIP3 palmitoylation (pharmacologically using 2-BP or genetically by mutating cysteines 47 and 48 to alanines) prevents its recruitment to mucin granules thereby affecting its function. It is important to note that mutation of KChIP3 palmitoylation sites did not completely prevent its localization to mucin granules. As suggested by Takimoto and colleagues palmitoylation may not act as a targeting or trafficking signal in KChIP protein family, but stabilize the association post membrane localization (Takimoto et al, 2002; Dunphy \& Linder, 1998). This would explain why KChIP3 palmitoylation mutant is still partially recruited to mucin granules. One possibility is that KChIP3 binds potassium or calcium channels in the mucin granule and palmitoylation is required for strengthening of this union. In the case of the mutant (KChIP3-AA), KChIP3 can still associate to mucin granules but, since it cannot be palmitoylated, the association is transient and cannot thus completely prevent mucin secretion.

\section{A) Mechanism of KChIP3 recruitment to mucin granules}

Based on the data shown here, together with our previous studies (Cantero-Recasens et al, 2019, 2018), we propose the following model for the regulation of KChIP3 association to mucin granules (Figure 4).

(1) Recruitment to granules. Cytosolic KChIP3 is palmitoylated by zDHHC3 and/or zDHHC4, which enhances its association with mucin granules.

(2) Brake for secretion. Once bound to mucin granules, KChIP3 inhibits baseline mucin secretion, and we postulate that it facilitates granule maturation by regulating ion channels at the membrane of mucin granules. 
(3) Release from granules. KChIP3 remains at the surface of mucin granules until intracellular calcium (as calcium oscillations generated by ryanodine receptors at the endoplasmic reticulum) reaches a threshold. At this point, KChIP3 binds calcium, what potentially triggers a change in its conformation, leading to its release from mucin granules.

(4) Mucin Secretion. KChIP3-free granules can fuse and release their contents to the extracellular medium to form the mucus layer.

\section{Conclusions}

In conclusion, KChIP3 recruitment to mucin granules is controlled by two opposite processes: palmitoylation and binding-to-calcium. Palmitoylation (possibly by zDHHC3 and/or zDHHC4) enhances the localization of KChIP3 to mucin granules, while binding to calcium releases it from granules. Dysregulation of any of these processes could lead to altered baseline mucin secretion (hypo or hypersecretion) that may end in pathological situations. Thus, zDHHC-dependent palmitoylation could be used as a pharmacological target to increase or decrease the activity of KChIP3, therefore controlling mucin secretion. 
$\underline{\text { Materials and Methods }}$

\section{Reagents and Antibodies}

All chemicals were obtained from Sigma-Aldrich (St. Louis, MO) except anti-MUC2 antibody clone 996/1 (RRID:AB_297837) (Abcam, Cambridge, UK), anti-MUC5AC antibody clone 45M1 (RRID: AB_934745) (Neomarkers, Waltham, MA) and antiKChIP3 antibody (RRID:AB_10608850) (Santa Cruz Biotechnology, Texas, USA). Secondary antibodies for immunofluorescence microscopy and dot blotting were from Life Technologies (ThermoFisher Scientific, Waltham, MA, USA).

\section{Cell lines}

HT29-18N2 cells (obtained from ATCC) (RRID:CVCL 5942) were tested for mycoplasma contamination with the Lookout mycoplasma PCR detection kit (SigmaAldrich, St. Louis, MO). Mycoplasma negative HT29-18N2 cells were used for the experiments presented here.

\section{Generation of rescue constructs KChIP3-WT and KChIP3-AA}

KCNIP3 shRNA targeting sequence (5'-GTGGAGAGGTTCTTCGAGA-3') from Lentilox 3.1 plasmid containing $K C N I P 3-$ WT was changed to a shRNA resistant sequence (5' GTTGAAAGATTTTTTGAAA-3') by Gibson assembly (Gibson et al, 2009) and restriction enzymes (NheI and AgeI). KChIP3-AA (rAA) construct was generated from KChIP3-WT (rWT) by site directed mutagenesis (Cysteines 45 and 46 to Alanines: 5'-TGCTGC-3' to 5'-GCCGCC-3' as shown in Figure 2A) (adapted protocol from (Zhang et al, 2009)).

\section{Generation of stable cell lines (rescue cell lines)}

HEK293T cells (ATCC, negative for mycoplasma) were co-transfected with the plasmid, VSV-G, pPRE (packaging) and REV by $\mathrm{Ca}^{2+}$ phosphate to produce lentiviruses. $48 \mathrm{hr}$ post transfection, the secreted lentivirus was collected, filtered and directly added to HT29-18N2 cells. Stably infected HT29-18N2 cells with the different constructs were sorted for GFP signal by FACS.

\section{Quantitative real-time PCR (RT-qPCR)}

Undifferentiated HT29-18N2 control cells and differentiated HT29-18N2 control, KChIP3-KD, KChIP3-GFP, rKChIP3-WT (rWT) and rKChIP3-AA (rAA) cells were lysed and total RNA extracted with the RNeasy extraction kit (Qiagen, Netherlands). cDNA was synthesized with Superscript III (Invitrogen). Primers for each gene (sequence shown below, Table 1) were designed using Primer-BLAST (NCBI) (Ye et $a l, 2012)$, limiting the target size to $150 \mathrm{bp}$ and the annealing temperature to $60^{\circ} \mathrm{C}$. To determine expression levels of the different genes, quantitative real-time PCR was performed with Light Cycler 480 SYBR Green I Master (Roche, Switzerland) according to manufacturer's instructions.

Table 1. Primer sequences used for detecting mRNA for the respective genes

\begin{tabular}{|l|l|c|}
\hline \multicolumn{1}{|c|}{ Gene } & Forward primer (5' $\mathbf{- 3}$ ') & Reverse primer (5' $\mathbf{- 3}$ ') \\
\hline $\begin{array}{l}\text { KCNIP3 } \\
\text { endogen }\end{array}$ & ACCCTCCTTCTTGCTAAGTGGT & AGGCTGGCAACAGTTTTCTTCA \\
\hline $\begin{array}{l}\text { KCNIP3 } \\
\text { exogen }\end{array}$ & ACCCTCCTTCTTGCTAAGTGGT & AGGCTGGCAACAGTTTTCTTCA \\
\hline
\end{tabular}




\begin{tabular}{|l|l|l|}
\hline GAPDH & GAAGGTGAAGGTCGGAGTCAAC & CATCGCCCCACTTGATTTTGGA \\
\hline HPRT1 & CCTGCTTCTCCTCAGCTTCAG & ACACCCTTTCCAAATCCTCAGC \\
\hline TBP & CGGCTGTTTAACTTCGCTTC & CAGACGCCAAGAAACAGTGA \\
\hline zDHHC2 & GTGTTCATCACCCTCCTGCTC & CCATCAGGCACACAACTTGTTC \\
\hline zDHHC3 & CCACTGCAGTGTTTGTAAGCGG & GAGAGGAGCTGCACTTTGTCC \\
\hline zDHHC4 & GGGAGGAGCACAGATATTTTCCTG & GGTGTGGTTTCTCGTATGGAAAAGG \\
\hline zDHHC13 & GTAATTGGGCCAGAACCAACTG & GGTGTTTCTCCCTTAACATTCTGG \\
\hline zDHHC14 & CGCCTTCGACTGTCCGTACC & $\begin{array}{l}\text { GGTGCCGTTTGCGATATCTAT } \\
\text { TTGCC }\end{array}$ \\
\hline zDHHC15 & CTCTGCCTGGTGACTGTTTTG & GTGTAGGACAAGTGGAACTTCTGG \\
\hline zDHHC17 & GCCCAGAATATCAAGGGCGAAT & GTACTGTAGCCCAAACACCACC \\
\hline zDHHC20 & CTCTGCGTGTGAAAGACCGTT & GATGCGCCCGATCAGGTTTAAT \\
\hline
\end{tabular}

\section{Differentiation of HT29-18N2 cells}

HT29-18N2 cells were differentiated to goblet cells as described previously (Mitrovic et al, 2013) Briefly, cells were seeded in complete growth medium (DMEM complemented with $10 \%$ FCS and $1 \% \mathrm{P} / \mathrm{S}$ ), and the following day (Day zero: D-0), the cells were placed in PFHMII protein free medium (GIBCO, ThermoFisher Scientific, Waltham, MA, USA). After 3 days (D-3), medium was replaced with fresh PFHMII medium and cells grown for 3 additional days. At day 6 (D-6) cells were trypsinized and seeded for the respective experiments in complete growth medium followed by incubation in PFHMII medium at day 7 (D-7). All experimental procedures were performed at day 9 (D-9).

\section{Mucin Secretion Assay}

HT29-18N2 cells were differentiated for 6 days and then split into 6-well plates. After one day (D-7), medium was exchanged with fresh PFHMII medium and cells grown for 2 more days. On D-9, cells were washed with isotonic solution containing: 140 $\mathrm{mM} \mathrm{NaCl}, 2.5 \mathrm{mM} \mathrm{KCl}, 1.2 \mathrm{mM} \mathrm{CaCl}_{2}, 0.5 \mathrm{mM} \mathrm{MgCl} 2,5 \mathrm{mM}$ glucose, and $10 \mathrm{mM}$ HEPES (305 mosmol/l, pH 7.4 adjusted with Tris); and then treated with vehicle (baseline secretion) or $100 \mu \mathrm{M}$ ATP (stimulated secretion) for $30 \mathrm{~min}$ at $37^{\circ} \mathrm{C}$. In order to inhibit zDHHC-dependent palmitoylation, cells were treated with $100 \mathrm{nM} 2$ $\mathrm{BP}$ or vehicle during the secretion assay. After $30 \mathrm{~min}$ at $37^{\circ} \mathrm{C}$, extracellular medium was collected and centrifuged for $5 \mathrm{~min}$ at $800 \mathrm{xg}$ at $4^{\circ} \mathrm{C}$. Cells were washed $2 \mathrm{X}$ in PBS and lysed in $2 \%$ Triton X-100/PBS for $1 \mathrm{hr}$ at $4^{\circ} \mathrm{C}$ and centrifuged at $14000 \mathrm{xg}$ for $15 \mathrm{~min}$.

\section{Dot Blot analysis}

Extracellular medium and cell lysates were spotted on nitrocellulose membranes $(0.45$ $\mu \mathrm{m})$ using Bio-Dot Microfiltration Apparatus (Bio-Rad, California, USA) (manufacturer's protocol) and membranes were incubated in blocking solution (5\% BSA/0.1\% Tween/PBS) for $1 \mathrm{hr}$ at room temperature. The blocking solution was removed and the membranes were incubated with an anti-MUC5AC antibody diluted 1:2000 or the anti-MUC2 antibody diluted 1:4000 in blocking solution, overnight at $4^{\circ} \mathrm{C}$. Membranes were then washed in $0.1 \%$ Tween/PBS and incubated with a donkey anti-mouse or anti-rabbit HRP coupled antibody (Life Technologies) for $1 \mathrm{hr}$ at room temperature. For the detection of $\beta$-actin and KChIP3, cell lysates were separated on SDS-PAGE, transferred to nitrocellulose membranes and processed as described for the dot blot analysis using the anti-B-actin (RRID:AB_476692), anti-KChIP3 (RRID:AB_10608850) or anti-GFP antibody (RRID:AB_390913) at a dilution of $1: 5000,1: 500$ and $1: 1000$ in 5\% BSA/0.1\% Tween/PBS, respectively. Membranes 
were washed and imaged with LI-COR Odyssey scanner (resolution $=84 \mu \mathrm{m})$ (LICOR, Nebraska, USA). Quantification was performed with ImageJ (FIJI, version 2.0.0-rc-43/1.51 g) (Schindelin et al, 2012). The number of experiments was greater than three for each condition, and each experiment was done in triplicates.

\section{KChIP3 and MUC5AC colocalization analysis}

Differentiated HT29-18N2 (Control, KChIP3-GFP, rWT or rAA) cells were grown on coverslips and on the day of the experiments cells were treated with $100 \mathrm{nM} 2-\mathrm{BP}$ or vehicle for $30 \mathrm{~min}$ at $37^{\circ} \mathrm{C}$. Next, to visualize MUC5AC and KChIP3 colocalization, differentiated HT29-18N2 cells were washed two times, at room temperature, with PBS for $5 \mathrm{~min}$. The cells were then permeabilized by incubation in a buffer (IB) containing $20 \mathrm{mM}$ HEPES pH 7.4, $110 \mathrm{mM}$ KOAc (Potassium acetate), $2 \mathrm{mM}$ $\mathrm{MgOAc}$ (Magnesium acetate) and $0.5 \mathrm{mM}$ EGTA (adapted from (Lorenz et al, 2008)) with $0.001 \%$ digitonin for $5 \mathrm{~min}$ on ice, followed by washing for $7 \mathrm{~min}$ on ice with the same buffer without detergent. Cells were fixed in 4\% paraformaldehyde for 15 min, further permeabilized for 5 min with $0.001 \%$ digitonin in IB and blocked with $4 \% \mathrm{BSA} / \mathrm{PBS}$ for $15 \mathrm{~min}$. The anti-MUC5AC antibody was then added to the cells at 1:5000 in $4 \% \mathrm{BSA} / \mathrm{PBS}$ overnight at $4{ }^{\circ} \mathrm{C}$; anti-GFP antibody was added to the cells at 1:500 in $4 \% \mathrm{BSA} / \mathrm{PBS}$ overnight at $4^{\circ} \mathrm{C}$. After $24 \mathrm{hr}$, cells were washed with PBS and incubated for $1 \mathrm{hr}$ at room temperature with a donkey anti-rabbit Alexa Fluor 555 (for GFP), anti-mouse Alexa Fluor 647 (for MUC5AC) (Life Technologies), diluted at 1:1000 in 4\% BSA/PBS, and DAPI (1:20000). Finally, cells were washed in PBS and mounted in FluorSave Reagent (Calbiochem, Billerica, MA). Images were acquired using an inverted Leica SP8 confocal microscope with a 63x Plan Apo NA 1.4 objective and analyzed using ImageJ ((FIJI, version 2.0.0-rc-43/1.51 g) (Schindelin et $a l, 2012)$. For detection of the respective fluorescence emission, the following laser lines were applied: DAPI, $405 \mathrm{~nm}$; and Alexa Fluor 555, $561 \mathrm{~nm}$; Alexa Fluor 647, $647 \mathrm{~nm}$. Two-channel colocalization analysis was performed using ImageJ, and the Manders' correlation coefficient was calculated using the plugin JaCop (Bolte \& Cordelières, 2006).

\section{Online analysis}

All zDHHC were searched in Uniprot database (https://www.uniprot.org/) and in the expression atlas from EMBL-EBI (https://www.ebi.ac.uk/gxa/home), focusing on the data from "A deep proteome and transcriptome abundance atlas of 29 healthy human tissues" (Wang et al, 2019) (expression level cutoff: 0).

\section{Statistical analysis}

All data are means \pm SEM. In all cases a D'Agostino- Pearson omnibus normality test was performed before any hypothesis contrast test. Statistical analysis and graphics were performed using GraphPad Prism 6 (RRID:SCR_002798) or SigmaPlot 10 (RRID:SCR_003210) software. For data that followed normal distributions, we applied either Student's t test or one-way analysis of variance (ANOVA) followed by Tukey's post hoc test. For data that did not fit a normal distribution, we used MannWhitney's unpaired t test and nonparametric ANOVA (Kruskal-Wallis) followed by Dunn's post hoc test. Criteria for a significant statistical difference were: ${ }^{*} \mathrm{p}<0.05$; $* * \mathrm{p}<0.01$.

\section{Acknowledgments}


We thank all members of the Malhotra Lab for valuable discussions. Cell sorting experiments were carried out by the joint CRG/UPF FACS Unit at Parc de Recerca Biomèdica de Barcelona (PRBB). Fluorescence microscopy was performed at the Advanced Light Microscopy Unit at the CRG, Barcelona. V.Malhotra is an Institució Catalana de Recerca i Estudis Avançats professor at the Centre for Genomic Regulation. This work was funded by grants from the Spanish Ministry of Economy and Competitiveness (BFU2013-44188-P to VM) and FEDER Funds. We acknowledge support of the Spanish Ministry of Economy and Competitiveness, through the Programmes "Centro de Excelencia Severo Ochoa 2013- 2017" (SEV2012-0208 \& SEV-2013-0347) and Maria de Maeztu Units of Excellence in R\&D (MDM-2015- 0502). This work reflects only the authors' views, and the EU Community is not liable for any use that may be made of the information contained therein.

\section{$\underline{\text { Figure Legends }}$}

Figure 1. 2-BP prevents KChIP3 function.

A) Control (black) and KChIP3 overexpressing cells (KChIP3-GFP) (red) were differentiated and incubated for $30 \mathrm{~min}$ at $37^{\circ} \mathrm{C}$ with vehicle or $100 \mathrm{nM} 2-\mathrm{BP}$ in absence (baseline) or presence of $100 \mu \mathrm{M}$ ATP (stimulated). Secreted MUC5AC was collected and dot blotted with an anti-MUC5AC antibody. Data were normalized to actin levels. The y-axis represent normalized values relative to the values of untreated control cells. B) Differentiated KChIP3-GFP cells were treated for $30 \mathrm{~min}$ at $37^{\circ} \mathrm{C}$ with vehicle or $100 \mathrm{nM} 2-\mathrm{BP}$ and then were processed for cytosolic washout. After fixation and permeabilization, samples were analyzed by immunofluorescence microscopy with an anti-KChIP3 (KChIP3, red) anti-MUC5AC (MUC5AC, green) and DAPI (blue). Images represent a single plane (xy) and an orthogonal view of each channel (xz). Scale bar $=5 \mu \mathrm{m}$. C) Colocalization between KChIP3 and MUC5AC was calculated from immunofluorescence images by Manders' coefficient using FIJI. Average values +- SEM are plotted as scatter plot with bar graph. The y-axis represents Manders' coefficient of the fraction of KChIP3 overlapping with MUC5AC. ${ }^{*} \mathrm{p}<0.05, * * \mathrm{p}<0.01$.

Figure 2. Generation of rescue cell lines.

A) Sequence alignment of KChIP3-WT with KChIP3-AA, which has both cysteines 47 and 48 (in bold) mutated into Alanines. Top line contains the nucleotide sequence, while lower line shows the protein sequence (aminoacids represented in three letter code). B) KCNIP3 endogenous (left plot) and total (right plot) RNA levels normalized to values of housekeeping genes GAPDH or HPRT1, respectively, from control (Ctrl), KChIP3 KD (K3 KD), KChIP3-GFP (K3 GFP), rescue KChIP3-WT (rWT) and rescue KChIP3-AA (rAA) differentiated cells. mRNA levels are represented as relative value compared to control cells. Results are plotted as scatter plot with bar graph showing average values \pm SEM $(\mathrm{N}>3)$. C) Cell lysates from control, rescue KChIP3-WT (rWT), rescue KChIP3-AA (rAA) and KChIP3-GFP (K3 GFP) differentiated cells were analyzed by western blot with an anti-KChIP3 and an antiGFP antibody to test expression levels. Actin was used as a loading control. ${ }^{*} \mathrm{p}<0.05$, $* * \mathrm{p}<0.01$.

Figure 3. Effect of KChIP3 palmitoylation mutant on mucin secretion. 
A) Secreted MUC5AC from differentiated control (black circles), KChIP3-KD (red circles), rKChIP3-WT (green circles) and rKChIP3-AA (yellow circles) cells that were incubated for $30 \mathrm{~min}$ at $37^{\circ} \mathrm{C}$ in the absence of external stimuli. Data were normalized to intracellular actin levels. The y-axis represents normalized values relative to the values of untreated control cells. B) Secreted MUC2 from differentiated control (black circles), KChIP3-KD (red circles), rKChIP3-WT (green circles) and rKChIP3-AA (yellow circles) cells that were incubated for $30 \mathrm{~min}$ at $37^{\circ} \mathrm{C}$ in the absence of external stimuli. Data were normalized to intracellular actin levels. The yaxis represents normalized values relative to the values of untreated control cells. C) Differentiated rKChIP3-WT and rKChIP3-AA cells were processed for cytosolic washout at basal conditions. After fixation and permeabilization, samples were analyzed by immunofluorescence microscopy with an anti-KChIP3 (KChIP3, red), anti-MUC5AC antibody (MUC5AC, green) and DAPI (blue). Images represent a single plane (xy) and an orthogonal view of each channel (xz). Scale bar $=5 \mu \mathrm{m}$. D) Colocalization between KChIP3 and MUC5AC was calculated from immunofluorescence images by Manders' coefficient using FIJI. Average values \pm SEM are plotted as scatter plot with bar graph. The y-axis represents Manders' coefficient of the fraction of KChIP3 overlapping with MUC5AC. *p<0.05, $* * \mathrm{p}<0.01$, n.s. not statistically significant.

\section{Figure 4. Model for KChIP3 recruitment to mucin granules.}

Cytosolic KChIP3 is recruited to mucin granules by a process involving palmitoylation (i). Once associated to mucin granules, KChIP3 blocks the release of their contents. KChIP3 stays on granules until calcium oscillations coming from ER (via ryanodine receptors) reach certain threshold and KChIP3 binds calcium. This binding promotes a conformational change in KChIP3 that leads to its release from mucin granules (ii) and therefore baseline mucin secretion can occur. Abbreviations: Palm.: Palmitoylation, RYR: Ryanodine Receptors, ER: Endoplasmic Reticulum.

Figure S1. zDHHC3 and zDHHC4 are upregulated in differentiated HT29-18N2 cells.

A) Expression levels and cellular location of zDHHCs in different human tissues according to Uniprot database and EMBL-EBI expression atlas (A deep proteome and transcriptome abundance atlas of 29 healthy human tissues (Wang et al, 2019)). In red those zDHHCs not localized at the Golgi. Black squares highlight colon (C.) and lungs (L.). B) RNA levels of zDHHC2, 3, 4, 13, 14, 15, 17 and 20 in undifferentiated (Undiff.) and differentiated (Diff.) HT29-18N2 cDNA were analyzed by agarose gel. C) zDHHC2, zDHHC3, zDHHC4, zDHHC7, zDHHC13, zDHHC14, zDHHC17 RNA levels from undifferentiated (UD) and differentiated (DF) HT29-18N2 by quantitative real-time PCR normalized to values of the housekeeping gene LTP. mRNA levels of each gene are represented as relative value compared to UD cells. Results are average values \pm SEM $(\mathrm{N} \geq 3)$. Abbreviations: Cell loc.: cellular localization, GA: Golgi apparatus, En: Endosomes, ER: Endoplasmic reticulum, PM: Plasmamembrane, A.T.: adipose tissue, A.G.: adrenal gland, B.M.: bone marrow, B.: brain, C.: colon, D.: duodenum, E.: endometrium, ES.: esophagus, F.T.: fallopian tube, G.B.: gallbladder, H.: heart, K.: kidney, L.: liver, LG.: lung, L.N.: lymph node, OV: ovary, P.: pancreas, P.G.: pituitary gland, PL.: placenta, PR.: prostate, R.: rectum, S.G.: salivary gland, S.I.: small intestine, S.M.: smooth muscle, SP.: spleen, ST.: stomach, T.: testis, TH.: thyroid, TN.: tonsil, U.B.: urinary bladder, V.A.: 
vermiform appendix, UD: Undifferentiated HT29-18N2, DF: Differentiated HT2918N2, C-: Negative control. *p<0.05

\section{$\underline{\text { References }}$}

Adler KB, Tuvim MJ \& Dickey BF (2013) Regulated Mucin Secretion from Airway Epithelial Cells. Front Endocrinol (Lausanne) 4

Birchenough GMH, Johansson MEV, Gustafsson JK, Bergström JH \& Hansson GC (2015) New developments in goblet cell mucus secretion and function. Mucosal Immunol 8: 712-719

Bolte S \& Cordelières FP (2006) A guided tour into subcellular colocalization analysis in light microscopy. J Microsc 224: 213-232

Buxbaum JD, Choi EK, Luo Y, Lilliehook C, Crowley AC, Merriam DE \& Wasco W (1998) Calsenilin: a calcium-binding protein that interacts with the presenilins and regulates the levels of a presenilin fragment. Nat Med 4: 1177-1181

Cantero-Recasens G, Butnaru CM, Brouwers N, Mitrovic S, Valverde MA \& Malhotra V (2019) Sodium channel TRPM4 and sodium/calcium exchangers (NCX) cooperate in the control of $\mathrm{Ca} 2+$-induced mucin secretion from goblet cells. J Biol Chem 294: 816-826

Cantero-Recasens G, Butnaru CM, Valverde MA, Naranjo JR, Brouwers N \& Malhotra V (2018) KChIP3 coupled to Ca2+ oscillations exerts a tonic brake on baseline mucin release in the colon. Elife 7

Carrión AM, Link WA, Ledo F, Mellström B \& Naranjo JR (1999) DREAM is a Ca2+-regulated transcriptional repressor. Nature 398: 80-84

Cone RA (2009) Barrier properties of mucus. Adv Drug Deliv Rev 61: 75-85

Dunphy JT \& Linder ME (1998) Signalling functions of protein palmitoylation. Biochim Biophys Acta 1436: 245-261

Ernst AM, Syed SA, Zaki O, Bottanelli F, Zheng H, Hacke M, Xi Z, Rivera-Molina F, Graham M, Rebane AA, et al (2018) S-Palmitoylation Sorts Membrane Cargo for Anterograde Transport in the Golgi. Dev Cell 47: 479-493.e7

Evans CM \& Koo JS (2009) Airway mucus: the good, the bad, the sticky. Pharmacol Ther 121: 332-348

Fahy JV \& Dickey BF (2010) Airway Mucus Function and Dysfunction. N Engl J Med 363: 2233-2247

Fukata M, Fukata Y, Adesnik H, Nicoll RA \& Bredt DS (2004) Identification of PSD95 palmitoylating enzymes. Neuron 44: 987-996

Gibson DG, Young L, Chuang R-Y, Venter JC, Hutchison CA \& Smith HO (2009) Enzymatic assembly of DNA molecules up to several hundred kilobases. Nat Methods 6: 343-345 
Jennings BC, Nadolski MJ, Ling Y, Baker MB, Harrison ML, Deschenes RJ \& Linder ME (2009) 2-Bromopalmitate and 2-(2-hydroxy-5-nitro-benzylidene)benzo[b]thiophen-3-one inhibit DHHC-mediated palmitoylation in vitro. $J$ Lipid Res 50: 233-242

Lemonidis K, Gorleku OA, Sanchez-Perez MC, Grefen C \& Chamberlain LH (2014) The Golgi S-acylation machinery comprises zDHHC enzymes with major differences in substrate affinity and S-acylation activity. Mol Biol Cell 25: 3870-3883

Lorenz H, Hailey DW \& Lippincott-Schwartz J (2008) Addressing membrane protein topology using the fluorescence protease protection (FPP) assay. Methods $\mathrm{Mol}$ Biol 440: 227-233

Mitrovic S, Nogueira C, Cantero-Recasens G, Kiefer K, Fernández-Fernández JM, Popoff J-F, Casano L, Bard FA, Gomez R, Valverde MA, et al (2013) TRPM5-mediated calcium uptake regulates mucin secretion from human colon goblet cells. Elife 2: e00658

Osawa M, Dace A, Tong KI, Valiveti A, Ikura M \& Ames JB (2005) Mg2+ and Ca2+ differentially regulate DNA binding and dimerization of DREAM. $J$ Biol Chem 280: 18008-18014

Pelaseyed T, Bergström JH, Gustafsson JK, Ermund A, Birchenough GMH, Schütte A, van der Post S, Svensson F, Rodríguez-Piñeiro AM, Nyström EEL, et al (2014) The mucus and mucins of the goblet cells and enterocytes provide the first defense line of the gastrointestinal tract and interact with the immune system. Immunol Rev 260: 8-20

Perez-Vilar J (2008) Formation of mucin granules. In The Golgi Apparatus: State of the art 110 years after Camillo Golgi's discovery, Mironov AA \& Pavelka M (eds) pp 535-562. Vienna: Springer Vienna

Rocks O, Gerauer M, Vartak N, Koch S, Huang Z-P, Pechlivanis M, Kuhlmann J, Brunsveld L, Chandra A, Ellinger B, et al (2010) The palmitoylation machinery is a spatially organizing system for peripheral membrane proteins. Cell 141: 458-471

Schindelin J, Arganda-Carreras I, Frise E, Kaynig V, Longair M, Pietzsch T, Preibisch S, Rueden C, Saalfeld S, Schmid B, et al (2012) Fiji: an open-source platform for biological-image analysis. Nat Methods 9: 676-682

Takimoto K, Yang E-K \& Conforti L (2002) Palmitoylation of KChIP splicing variants is required for efficient cell surface expression of Kv4.3 channels. $J$ Biol Chem 277: 26904-26911

Thornton DJ, Rousseau K \& McGuckin MA (2008) Structure and function of the polymeric mucins in airways mucus. Annu Rev Physiol 70: 459-486

Wang D, Eraslan B, Wieland T, Hallström B, Hopf T, Zolg DP, Zecha J, Asplund A, Li L, Meng C, et al (2019) A deep proteome and transcriptome abundance atlas of 29 healthy human tissues. Molecular Systems Biology 15: e8503 
Ye J, Coulouris G, Zaretskaya I, Cutcutache I, Rozen S \& Madden TL (2012) PrimerBLAST: a tool to design target-specific primers for polymerase chain reaction. BMC Bioinformatics 13: 134

Zhang B, Zhang X, An X, Ran D, Zhou Y, Lu J \& Tong Y (2009) An easy-to-use site-directed mutagenesis method with a designed restriction site for convenient and reliable mutant screening*. J Zhejiang Univ Sci B 10: 479-482

Zhu Y, Abdullah LH, Doyle SP, Nguyen K, Ribeiro CMP, Vasquez PA, Forest MG, Lethem MI, Dickey BF \& Davis CW (2015) Baseline Goblet Cell Mucin Secretion in the Airways Exceeds Stimulated Secretion over Extended Time Periods, and Is Sensitive to Shear Stress and Intracellular Mucin Stores. PLoS ONE 10: e0127267 
Figure 1

A.

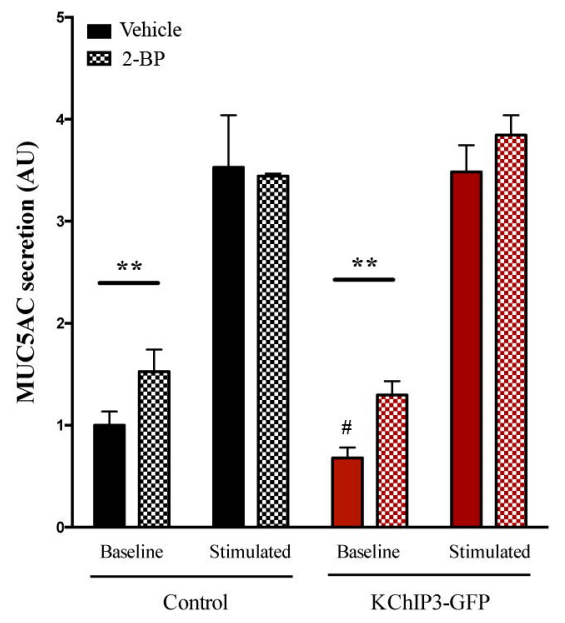

B.

KChIP3

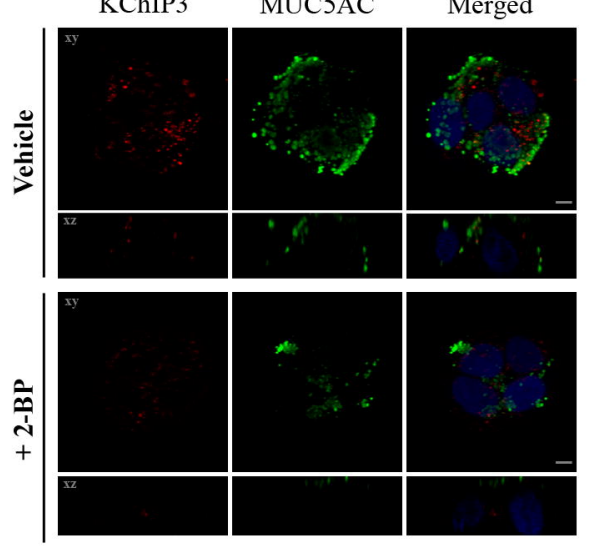

C.

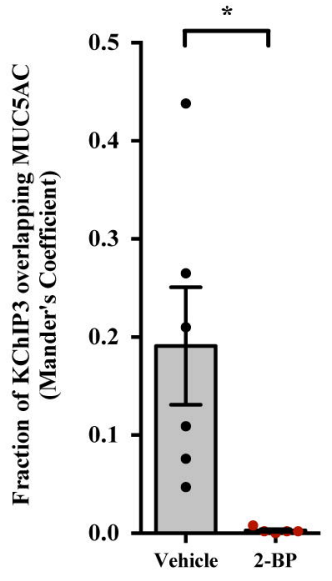




\section{Figure 2}

A.

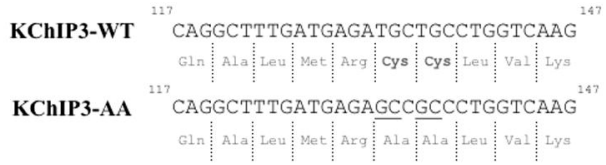

B.
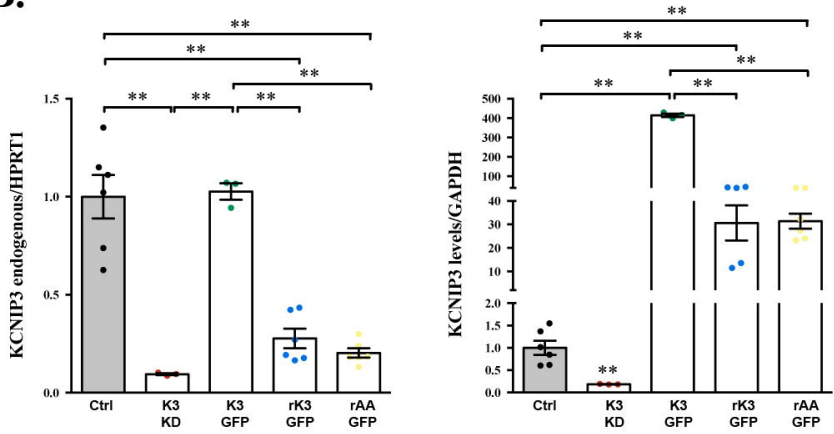

C.

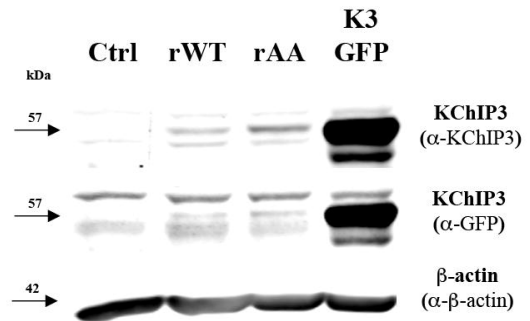


Figure 3

A.

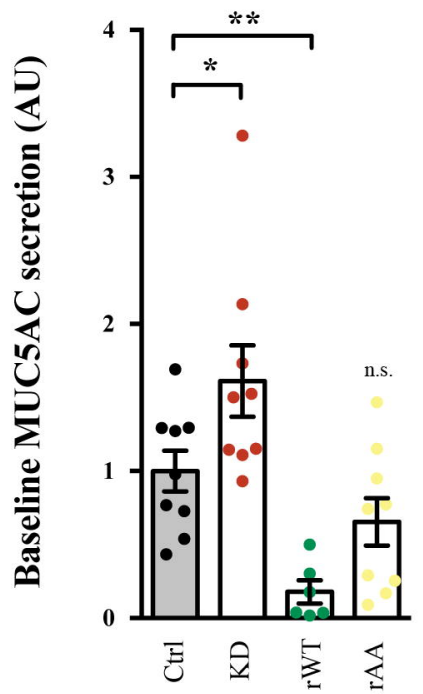

B.

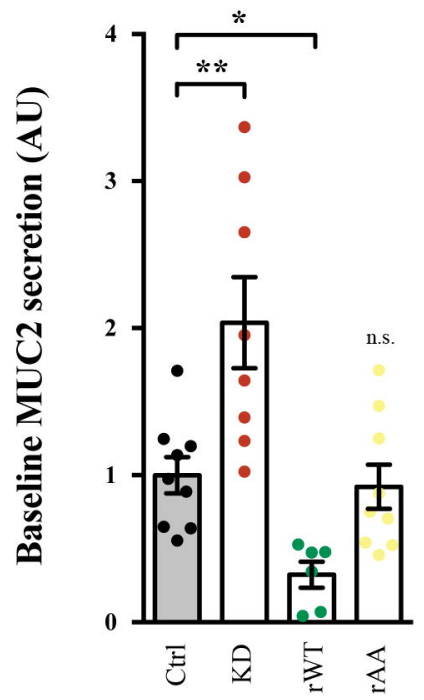

C.

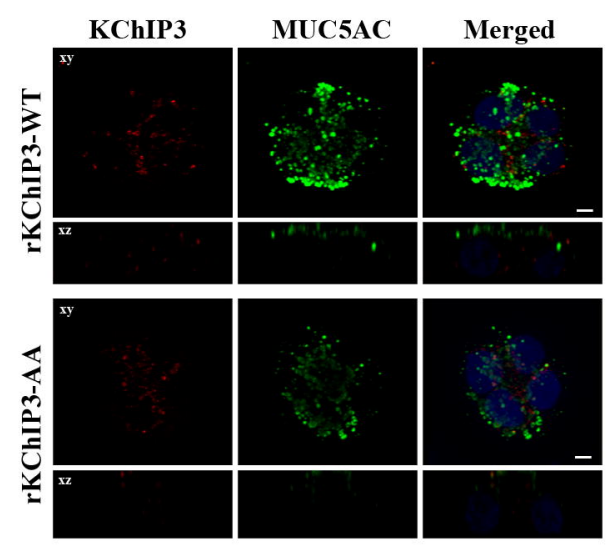

D.

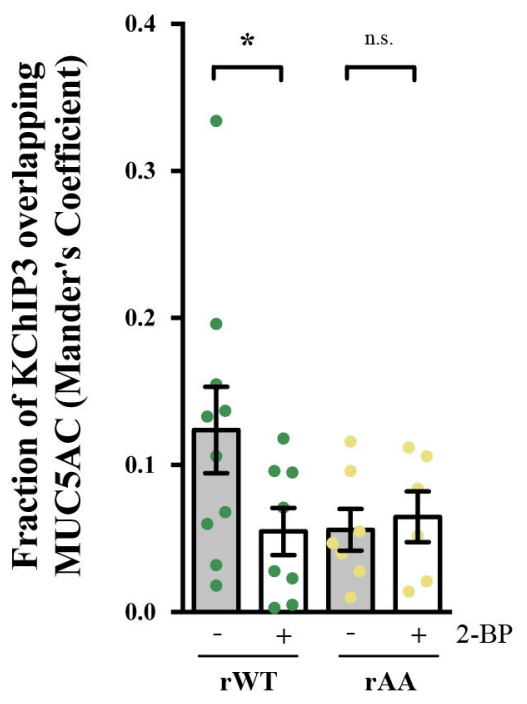


Figure 4
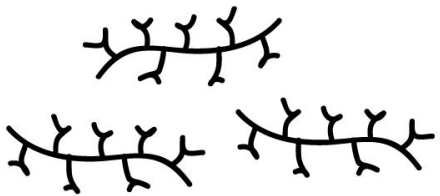

Cytosol
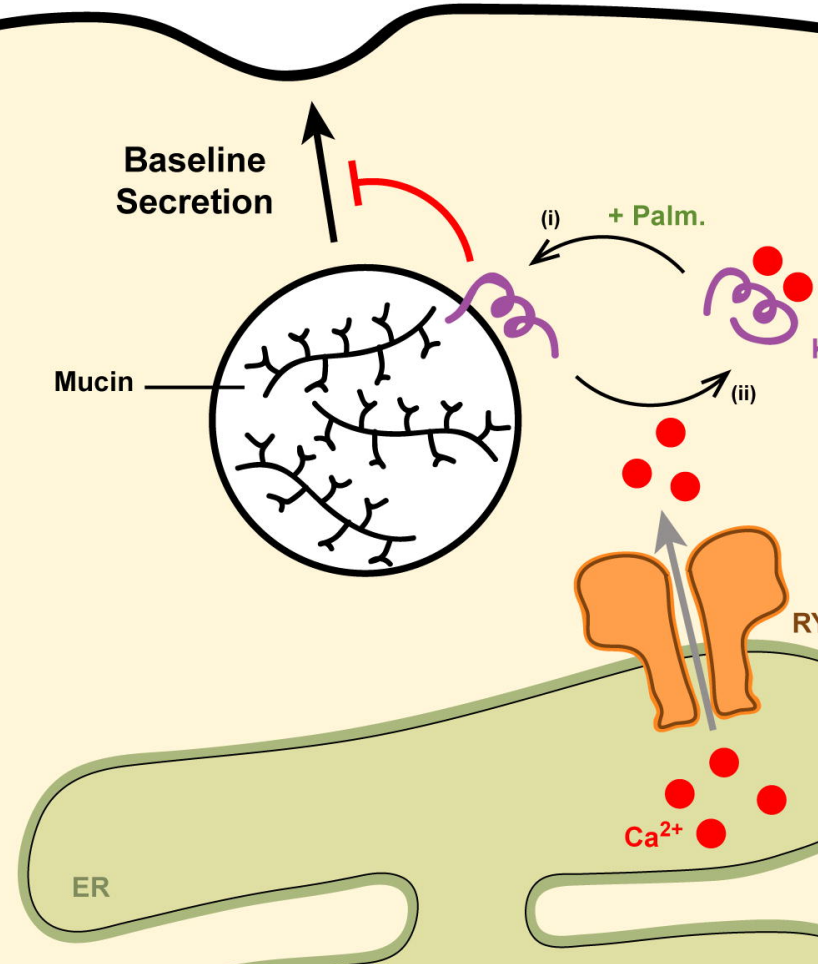

KChIP3

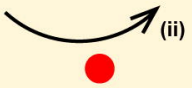

RYR

$\mathrm{Ca}^{2+}$

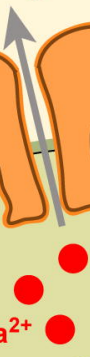

